\title{
Replication-selective adenoviruses as oncolytic agents
}

\author{
Carla Heise ${ }^{1}$ and David H. Kirn ${ }^{2}$ \\ ${ }^{1}$ Chiron Corporation, Emeryville, California 94608, USA \\ ${ }^{2}$ Imperial Cancer Research Fund and the Imperial College School of Medicine, Program for Viral and Genetic \\ Therapy of Cancer, Hammersmith Hospital, London WI2 ONN, United Kingdom
}

Address correspondence to: David Kirn, Imperial Cancer Research Fund, Molecular Oncology Unit, Imperial College School of Medicine, Hammersmith Hospital, London WI2 ONN, United Kingdom. Phone: 44-181-383-8584; Fax: 44-181-383-3258; E-mail: d.kirn@icrf.icnet.uk.

Adenovirus biology and cancer biology have progressed in a complementary fashion. For example, proteins encoded at the virus' E1 region were found to bind to the tumor suppressor proteins $\mathrm{p} 53$ and $\mathrm{pRB}$, and these interactions played an important role in characterizing the role of these tumor suppressors in virus-dependent and -independent transformation $(1,2)$. Perhaps, then, it is a natural extension of this history that human adenoviruses are now being developed as anticancer agents. Ironically, although advances in genetic engineering have permitted the development of replication-disabled adenoviruses as simple delivery vehicles for therapeutic genes (3-5), replication-competent wildtype strains were actually the first to be administered safely to patients (6). Now, nearly a half-century later, replication-competent adenoviruses are once again being studied as therapeutic agents (7-9). However, genetic engineering has now been used with the goal of maximizing tumor-selective replication.

Replication-selective adenoviruses have several appealing properties as biotherapeutic agents. Replication amplifies the input dose of the oncolytic virus and helps spread the agent to adjacent tumor cells (9). In addition, adenoviral infection may generate an antitumoral immune response (10-12). Given the favorable attributes of both therapeutic genes and replication-

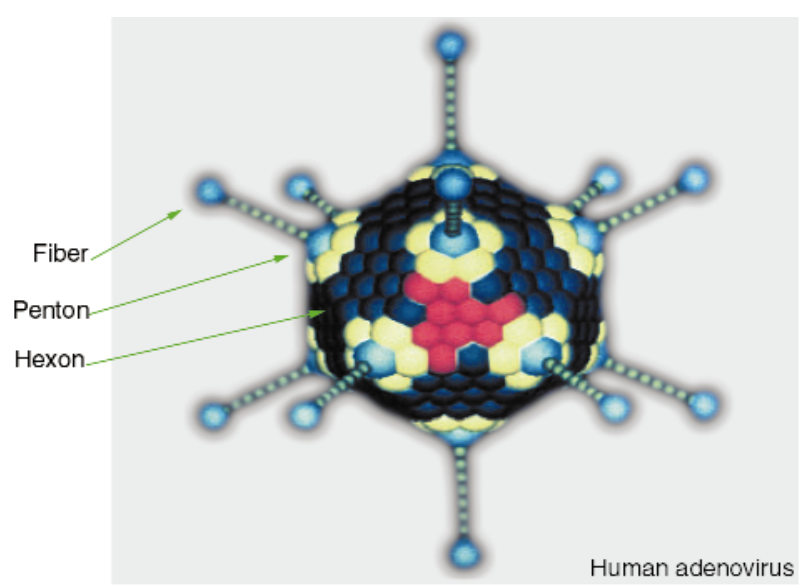

Figure 1

Structure of human adenovirus, indicating the arrangement of capsid proteins. Fiber and penton recognize cellular receptors that mediate endocytosis of the virus. competent viral approaches, it is likely that the best clinical success will eventually be achieved with adenoviruses armed with therapeutic genes, used to complement standard surgical, radio-, and chemotherapeutic approaches.

A number of efficacy, safety, and manufacturing issues need to be assessed when considering a virus species for development as an oncolytic therapy. First, by definition, the virus must replicate in and destroy human tumor cells. Rational engineering of the virus depends on a detailed understanding of viral genes modulating infection, replication, and pathogenesis. Since most solid human tumors have relatively low growth fractions, the virus should infect noncycling cells. In addition, receptors for viral entry must be expressed on the target tumor(s) in patients. From a safety standpoint, the parental wildtype virus should cause, at worst, mild and readily controllable human diseases. Nonintegrating viruses have potential safety advantages, as well. A genetically stable virus is desirable from both safety and manufacturing standpoints. Finally, the virus must be amenable to high-titer production under Good Manufacturing Practices (GMP) guidelines for clinical studies. Human adenoviruses have these characteristics and are therefore excellent candidates for therapeutic development.

\section{Biology of human adenovirus}

All adenoviruses (Figure 1) have linear, double-stranded DNA genomes of approximately $38 \mathrm{kB}$ (13). The capsid is nonenveloped and is composed of the structural proteins hexon; penton, which binds the $\alpha_{v} \beta_{3}$ and $\alpha_{v} \beta_{5}$ integrins to allow virus internalization; and fiber, which binds the coxsackie and adenovirus receptor, CAR. The E3 region of the genome encodes several proteins that allow the virus to evade immune responses (14). The gp19 kD protein inhibits MHC-class I expression on the cell surface (i.e., avoidance of cytotoxic $\mathrm{T}$ lymphocyte-mediated killing) (15), and the E3 10.4/14.5 kD and $14.7 \mathrm{kD}$ proteins inhibit apoptosis mediated by FasL or tumor necrosis factor (TNF) $(12,14)$.

The adenovirus lifecycle (reviewed in ref. 16) proceeds through the following steps: (a) virus entry into the cell following CAR and integrin binding, (b) cell entry into S-phase, (c) suppression of host cell apoptosis, (d) blockade of cellular protein synthesis, (e) viral DNA replication, (f) viral structural protein synthesis, (g) virion assembly in the nucleus, (h) cell death, and (i) virus release. Adenovirus replication within a target 
Table 1

Potential mechanisms of antitumoral efficacy with replication-selective adenoviruses

\begin{tabular}{|c|c|}
\hline Mechanism & $\begin{array}{l}\text { Examples of adenoviral } \\
\text { genes modulating effect }\end{array}$ \\
\hline I. Direct cytotoxicity due to viral proteins & $\begin{array}{c}\text { E3 11.6kD } \\
\text { E4ORF4 }\end{array}$ \\
\hline \multicolumn{2}{|l|}{ II. Augmentation of antitumoral immunity } \\
\hline CTL infiltration, killing & E3 gp19kD* \\
\hline tumor cell death, antigen release & E3 $11.6 \mathrm{kD}$ \\
\hline immunostimulatory cytokine induction & E3 10.4/14.5, 14.7kD* \\
\hline antitumoral cytokine induction (e.g. TNF) & E3 $10.4 / 14.5,14.7 \mathrm{kD} *$ \\
\hline enhanced sensitivity to cytokines (e.g. TNF) & E1A \\
\hline III. Sensitization to chemotherapy & $\begin{array}{c}\text { Unknown } \\
\text { (? E1A, others) }\end{array}$ \\
\hline IV. Expression of exogenous therapeutic genes & NA \\
\hline
\end{tabular}

*Viral protein inhibits antitumoral mechanism. CTL, cytotoxic T-lymphocyte. TNF, tumor necrosis factor. NA, not applicable.

tumor cell can cause cell destruction by several mechanisms (Table 1). First, viral proteins, including the E3 11.6 adenovirus death protein (16) and E4ORF4 (17), that are expressed late in the course of infection are directly cytotoxic. Deletion of the corresponding genes significantly delays death of infected cells. In addition, expression of E1A early during the adenovirus lifecycle sensitizes cells to tumor necrosis factor (TNF)-mediated killing (18). This effect is inhibited by the E3 proteins 10.4/14.5 and 14.7; deletion of these E3 proteins leads to an increase in TNF expression in vivo and enhanced cell sensitivity to TNF. Finally, viral replication in and lysis of tumor cells induces cell-mediated immunity to tumor cells in model systems (R. Martuza, this series). Whether patients will benefit from a similar effect remains to be determined.

\section{Clinical trial results with wildtype adenovirus}

The knowledge that adenoviruses could eradicate a variety of tumor cells in vitro led to a clinical trial in the 1950s with 10 serotypes of wild-type adenoviruses in cervical cancer patients (6). Forty total treatments were administered to 30 patients including direct intratumoral injection, injection into the artery perfusing the tumor, or intravenous administration. When possible, the patients were treated with a serotype to which they had no neutralizing antibodies present. Corticosteroids were administered as nonspecific immunosuppressive agents in roughly half the cases.

No significant local or systemic toxicity was reported. This relative safety is notable given the lack of preexisting immunity to the serotype used and concomitant corticosteroid use in many patients. Some patients experienced a relatively mild viral syndrome that lasted 2 to 7 days and resolved spontaneously. Infectious adenovirus was recovered from the tumor in two-thirds of the patients for up to 17 days post-inoculation. Twothirds of the patients showed a "marked to moderate local tumor response" with necrosis and ulceration of the tumor. Unfortunately, the definition of a response is not reported, but no response occurred among the seven control patients treated with either virus-free tis- sue culture fluid or heat-inactivated virus. Neutralizing antibodies increased within 7 days after administration. Although the clinical benefit to these patients is unclear, and all patients eventually had tumor progression and died, this study demonstrated that wildtype adenoviruses can be safely administered to patients and can cause tumor necrosis in the face of an immune response.

Because this study did not include a systematic escalation of the viral dose, it did not identify a maximally tolerated dose or clarify the magnitude of the therapeutic index-a measure of the potency and selectivity of a treatment-for treatment with wildtype virus. Since all routes of administering the treatment expose normal tissue to live virus $(19,20)$, the clinical utility of any such agent will depend on its therapeutic index. A number of approaches to optimizing selectivity and potency are being pursued currently.

\section{Optimizing tumor-selective adenovirus replication}

Two broad approaches are currently being used to engineer tumor-selective adenovirus replication (Figure 2). The first is to limit the expression of the E1A gene product to tumor tissues through the use of tumor- or tissue-specific promoters. E1A stimulates S-phase entry and transactivates both viral and cellular genes that are critical for a productive viral infection (21). Tissue-or tumor-specific promoters can replace endogenous viral sequences in order to restrict viral replication to a particular target tissue. For example, the prostate-specific antigen gene (PSA) promoter/enhancer element has been inserted upstream of the $E 1 A$ gene; the result is that viral replication correlates with the level of PSA expression in a given cell (22). This virus, CN706 (Calydon Pharmaceuticals, Sunnyvale, California, USA), is currently in a phase I clinical trial of intratumoral injection for patients with locally recurrent prostate carcinoma. A second prostate-specific enhancer sequence has subsequently been inserted upstream of the $E 1 B$ region in the CN706 virus; the use of these two prostate-specific enhancer elements to drive separate early gene regions has led to improved selectivity over the first generation 
virus. A similar approach has been pursued by other groups using tissue-specific promoters to drive E1A expression selectively in specific carcinomas (e.g. alphafetoprotein, carcinoembryonic antigen, MUC-1).

A second strategy for optimizing tumor selectivity is to delete gene functions that are critical for efficient viral replication in normal cells but not in tumor cells. Many of the same critical regulatory proteins that are inactivated by viral gene products during adenovirus replication are also inactivated during carcinogenesis $(2$, $23,24)$. Because of this convergence, viral gene products that would ordinarily be required to inactivate a cellular protein become superfluous in cancer cells that have already lost the target protein $(25,26)$. For example, the function of the p53 tumor suppressor gene product is compromised both in tumors and in adenovirus-infected cells. A strain of adenovirus deleted for the $E 1 B-55 k D$ gene, dl1520 (ONYX-015, now known as CI-1042, ParkDavis Pharmaceuticals, Inc., division of Warner Lambert, Inc. Ann Arbor, Michigan, USA), is being developed for the treatment of tumors lacking p 53 function (2). Since the $\mathrm{E} 1 \mathrm{~B}-55 \mathrm{kD}$ gene product is responsible for p53-binding and inactivation, it was hypothesized that the deletion mutant would be unable to inactivate p53 in normal cells and would thus be unable to replicate efficiently. In contrast, cancer cells lacking functional p53 would be expected to be sensitive to viral replication and subsequent cytolysis. Similarly, the tumor suppressor $\mathrm{pRb}$, which is inhibited by the viral protein E1A, is frequently lost during tumorigenesis. Mutants in the pRB-binding domain of E1A are being evaluated for use against such tumors; these mutants demonstrate significantly greater potency than wild-type or other genedeleted adenoviruses both in culture and in vivo (26).

The p53-dependent specificity of dl1520 has been controversial. Bischoff et al. (27) initially reported that $p 53$ mutant tumor cells were destroyed in a replication- dependent fashion both in vitro and in vivo. Several groups subsequently showed that many tumor cell lines with normal $p 53$ gene sequences were also relatively sensitive to the effects of $d l 1520$ (28-31), but in light of the multiple mechanisms by which p53 function can be lost-in addition to genetic mutation-this finding is predictable. $d l 1520$ was significantly attenuated, relative to wild-type adenovirus, in most normal cell types tested $(25,30,32)$, and comparisons of cell lines that are identical except for $\mathrm{p} 53$ function show that the replication or cytopathic effects of $d l 1520$ are inhibited by functional p53 (ref. 27; I. Ganly and C. Heise, unpublished data). On the other hand, one cell line, U2OS, did not become significantly more sensitive to $d l 1520$ following transfection with dominant-negative p53 (32). It is clear that a background of other mutations in tumor cells will substantially influence the replication and/or cytopathic effects of $d l 1520$ and other adenoviruses.

Despite the intrinsic appeal of the strategy used by dl1520-exploiting common loss-of-function mutations in tumor cells to direct replication of mutant adenoviruses specifically to transformed cells-several potential hurdles must be considered. First, important viral functions may occur through redundant mechanisms, so alteration or deletion of a single viral gene may not abolish a given activity completely. $d l 1520$ is severely attenuated in normal endothelial and mammary epithelial cells, for example, but not in some other normal cells. Moreover, if a viral gene is deleted that confers only relatively minor survival advantages, the resulting mutant strain will be only partially attenuated for growth in normal host cells. Conversely, many adenoviral gene products are multifunctional; deletion of such a gene would result in loss of more than one function. Such a deletion mutant may be significantly attenuated, relative to wildtype adenovirus, even in tumor cells of the desired genotype. For example, the relative effi-

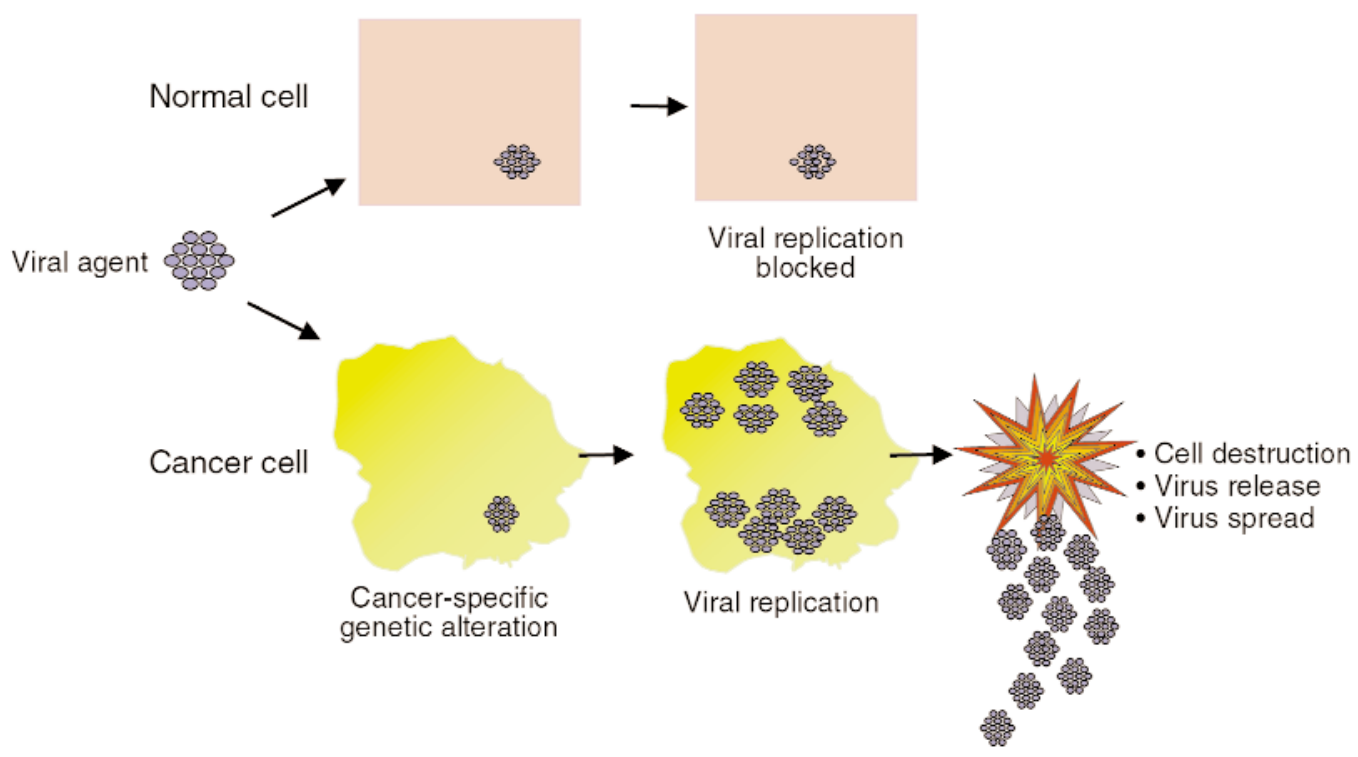

Figure 2

Selective killing of cancer cells with a replication-selective agent. Viral agents can be engineered to replicate in and to kill tumor cells specifically, leaving healthy cells unharmed. Several strategies are available for designing such agents. 
ciency of cell killing by $d l 1520$ and by wildtype adenovirus varies significantly between different p53 mutant cancer cell lines (34). In working with such genes, it may be useful to introduce subtle mutations, rather than deletions, to avoid introducing pleiotropic defects into the mutant virus. Finally, functional pathways in cancer cells are highly complex and can be inactivated through numerous mechanisms; analysis of a single gene sequence within a cancer cell is unlikely to accurately predict the efficacy of any viral mutant.

\section{Clinical development of $d l 1520$ (ONYX-015, now known as CI1042): a staged development approach} $d l 1520$ is a novel agent with a novel mechanism of action. We predicted that both toxicity and efficacy would depend on the intrinsic ability of a given tumor to replicate the virus, the location of the tumor to be treated (e.g. intracranial vs. peripheral) and the route of administration of the virus. In order to follow viral replication, antiviral immune responses and their relationship to antitumoral efficacy throughout clinical development, we used a staged clinical development approach. Intratumoral injection protocols were followed by trials of intracavitary instillation, intra-arterial infusion, and eventually intravenous administration. Patients with recurrent head and neck carcinomas were chosen as our initial study population because such tumors commonly carry p53 abnormalities and are usually accessible, even in an outpatient setting, for direct injection and biopsy. Since most patients suffer severe morbidity, and even mortality, from the local progression of these tumors, we hoped that local therapy might significantly improve their condition or prolong their survival.

Phase I and II testing has been completed for intratumoral injection of $d l 1520$ into recurrent and refractory head and neck carcinomas. Preliminary results, reported previously $(20,33,34)$, establish biological proof-of-concept for the replication-selective adenovirus approach: Most head and neck carcinoma biopsies showed selective intratumoral replication, followed in some cases by tumor necrosis and virus-induced cytopathic effects. Normal tissue adjacent to the injected tumor masses showed no significant toxicity. Importantly, neutralizing antibody titers at baseline did not appear to influence antitumoral activity or toxicity (33).

$d l 1520$ has also been tested in phase I and II trials for locally advanced pancreatic carcinoma, ovarian cancer, colorectal carcinoma, non-small cell lung carcinoma, and oral dysplasias, using a variety of systemic or local treatments to administer the virus. $d l 1520$ has been safely administered to over 200 patients, and a maximally tolerated dose has not been identified by any route of administration at doses up to $2 \times 10^{13}$ particles. As with the head and neck carcinoma data, biopsies from treated colorectal liver metastases showed that ONYX-015 had replicated, but, in other trials, no significant intratumoral replication was evident in ovarian or pancreatic carcinomas. Despite clear evidence of antitumoral activity in some patients (e.g. induction of necrosis), treatment with ONYX-015 as a single agent has not produced classically-defined objective tumor responses in patients. Therefore, $d l 1520$ is well-tolerated by numerous routes of administration, but as a single agent its potency has been limited.

\section{Enhancing the potency of adenovirus- based biotherapy}

In order to increase the potency of treatment, we combined $d l 1520$ with a conventional chemotherapeutic regimen [intravenous cisplatin and 5-fluorouracil (5-FU)] in a phase II head and neck cancer study. Response rates of injected tumors were superior to historical controls treated with chemotherapy alone; using combined therapy, $83 \%$ of tumors injected failed to progress within 6 months after therapy, compared to 25 to $30 \%$ of tumors treated with the same chemotherapy alone, historically (35). Patients with more than one tumor mass had one tumor injected with $d l 1520$ and secondary tumors were not injected; these noninjected tumors served as internal controls for the effects of chemotherapy alone. $d l 1520$ injected tumors had significantly better response rates and time-to-tumor-progression than the noninjected tumors. A colorectal liver metastasis trial also provided preliminary evidence that 5 -FU and $d l 1520$ can interact favorably (Tony Reid and D. Kirn, unpublished data). A randomized phase III trial comparing combination viral and chemotherapy versus chemotherapy alone in this patient population will begin this year.

An alternative approach to increasing the oncolytic activity of selectively replicating adenoviruses involves engineering these vectors to express exogenous therapeutic genes (see both T. Hermiston and D. Springer, this series). These approaches are clearly not mutually exclusive, and second-generation transgenic constructs may combine the advantages of direct local and systemic antitumoral activity with selective replication and specifically enhanced chemosensitivity in cancer cells. transformation by the adenovirus E1A proteins. Cell. 56:67-75.

2. Barker, D.D., and Berk, A.J. 1987. Adenovirus proteins from both E1B reading frames are required for transformation of rodent cells by viral infection and DNA transfection. Virology. 156:107-121.

3. Gomez-Manzano, C., et al. 1996. Adenovirus-mediated transfer of the p53 gene produces rapid and generalized death of human glioma cells via apoptosis. Cancer Res. 56:694-699.

4. Roth, J., and Cristiano, R.J. 1997. Gene therapy for cancer: what have we done and where are we going? J. Natl. Cancer Inst. 89:21-39.

5. Merritt, J., et al. 1997. Clinical phase I experience with INGN 201 (Adp53) in non-small cell lung cancer and head and neck cancer. Cancer Gene Ther. 4:S12. (Abst.)

6. Smith, R., Huebner, R.J., Rowe, W.P., Schatten, W.E., and Thomas, L.B. 1956. Studies on the use of viruses in the treatment of carcinoma of the cervix. Cancer. 9:1211-1218.

7. Kirn, D.H. 1996. Replicating oncolytic viruses: an overview. Expert Opinion on Investigational Drugs. 5:753-762.

8. Heise, C., Williams, A., Xue, S., Propst, M., and Kirn, D. 1999. Intravenous administration of ONYX-015, a selectively-replicating adenovirus, induces antitumoral efficacy. Cancer Res. 59:2623-2628.

9. Kirn, D.H., and McCormick, F. 1996. Replicating viruses as selective cancer therapeutics. Mol. Med. Today. 2:519-527.

10. Day, D.B., Zachariades, N.A., and Gooding, L.R. 1994. Cytolysis of adenovirus-infected murine fibroblasts by IFN-gamma-primed macrophages is TNF- and contact-dependent. Cell Immunol. 157:223-238.

11. Gooding, L.R. 1994. Regulation of TNF-mediated cell death and inflammation by human adenoviruses. Infect. Agents Dis. 3:106-115.

12. Shisler, J., Duerksen, H.P., Hermiston, T.M., Wold, W.S., and Gooding, L.R. 1996. Induction of susceptibility to tumor necrosis factor by E1A is dependent on binding to either $\mathrm{p} 300$ or $\mathrm{p} 105-\mathrm{Rb}$ and induction of DNA synthesis. J. Virol. 70:68-77.

13. Kirn, D. 1999. Selectively-replicating viuses as therapeutic agents against 
cancer. In: Cancer Gene Therapy. E. Lattime and S. Gerson, eds.,. Academic Press. San Diego, CA. 235-248

14. Dimitrov, T., et al. 1997. Adenovirus E3-10.4K/14.5K protein complex inhibits tumor necrosis factor-induced translocation of cytosolic phospholipase A2 to membranes. J. Virol. 71:2830-2837.

15. Hermiston, T.W., Tripp, R.A., Sparer, T., Gooding, L.R., and Wold, W.S 1993. Deletion mutation analysis of the adenovirus type 2 E3-gp19K protein: identification of sequences within the endoplasmic reticulum lumenal domain that are required for class I antigen binding and protection from adenovirus-specific cytotoxic T lymphocytes. J. Virol. 67:5289-5298.

16. Tollefson, A.E., Ryerse, J.S., Scaria, A., Hermiston, T.W., and Wold, W.S. 1996. The E3-11.6-kDa adenovirus death protein (ADP) is required for efficient cell death: characterization of cells infected with adp mutants. Virology. 220:152-162.

17. Branton, P. 1999. Early gene expression. In Adenoviruses: basic biology to gene therapy. P. Seth, editor. R.G. Landes, Austin, TX. 39-58.

18. Gooding, L.R. 1994. Regulation of TNF-mediated cell death and inflammation by human adenoviruses. Infect Agents Dis. 3:106-115.

19. Heise, C., Williams, A., Olesch, J., and Kirn, D. 1999. Efficacy of a replication-competent adenovirus (ONYX-015) following intratumoral injection: intratumoral spread and distribution effects. Cancer Gene Ther 6:499-504.

20. Ganly, I., et al. 1997. A phase I clinical trial with ONYX-015 (a selectively replicating adenovirus) administered by intratumoral injection in patients with recurrent head and neck cancer. Clin. Cancer Res. (In press).

21. Whyte, P., Ruley, H., and Harlow, E. 1988. Two regions of the adenovirus early region $1 \mathrm{~A}$ proteins are required for transformation. J. Virol. 62:257-265.

22. Rodriguez, R., et al. 1997. Prostate attenuated replication competent adenovirus (ARCA) CN706: a selective cytotoxic for prostate-specific antigen-positive prostate cancer cells. Cancer Res. 57:2559-2563.

23. Sherr, C.J. 1996. Cancer cell cycles. Science. 274:1672-1677.

24. Olson, D.C., and Levine, A.J. 1994. The properties of p53 proteins selected for the loss of suppression of transformation. Cell Growth Differ. 5:61-71.
25. Heise, C., et al. 1997. ONYX-015, an E1B gene-attenuated adenovirus, causes tumor-specific cytolysis and antitumoral efficacy that can be augmented by standard chemotherapeutic agents . Nat. Med. 3:639-645.

26. Kirn, D., Heise, C., Williams, M., Propst, M., and Hermiston, T. 1998. Adenovirus E1A CR2 mutants as selectively-replicating agents for cancer. Presented at Cancer Gene Therapy, meeting(R. Sobol and K. Scanlon, organizers). San Diego, Ca.

27. Bischoff, J.R., et al. 1996. An adenovirus mutant that replicates selectively in p53-deficient human tumor cells. Science. 274:373-376.

28. Heise, C., et al. 1997. ONYX-015, an E1B gene-attenuated adenovirus, causes tumor-specific cytolysis and antitumoral efficacy that can be augmented by standard chemotherapeutic agents. Nat. Med. 3:639-645.

29. Goodrum, F.D., and Ornelles, D.A. 1997. The early region 1B 55-kilodalton oncoprotein of adenovirus relieves growth restrictions imposed on viral replication by the cell cycle. J. Virol. 71:548-561.

30. Hall, A.R., Dix, B.R., O'Carroll, S.J., and Braithwaite, A.W. 1998. p53dependent cell death/apoptosis is required for a productive adenovirus infection [see comments]. Nat. Med. 4:1068-1072.

31. Turnell, A.S., Grand, R.J., and Gallimore, P.H. 1999. The replicative capacities of large E1B-null group A and group C adenoviruses are independent of host cell p53 status. J. Virol. 73:2074-2083.

32. Rothmann, T., Hengstermann, A., Whitaker, N.J., Scheffner, M., and zur Hausen, H. 1998. Replication of ONYX-015, a potential anticancer adenovirus, is independent of $\mathrm{p} 53$ status in tumor cells. J. Virol. 72:9470-9478.

33. Kirn, D., et al. 1998. A phase II trial of intratumoral injection with an E1B-deleted adenovirus, ONYX-015, in patients with recurrent, refractory head and neck cancer. Proc. Am. Soc. Clin. Oncol. 17:391a. (Abst.)

34. Kirn, D., Hermiston, T., and McCormick, F. 1998. ONYX-015: clinical data are encouraging. Nat. Med. 4:1341-1342.

35. Kirn, D.H., et al. 1999. A phase II trial of ONYX-015, a selectively-replicating adenovirus, in combination with cisplatin and 5 -fluorouracil in patients with recurrent head and neck cancer. Proc. Am. Soc. Clin. Oncol. 18:1505. 